\title{
ASYMPTOTIC BEHAVIOUR OF CRITICAL CONTROLLED BRANCHING PROCESSES WITH RANDOM CONTROL FUNCTIONS
}

\author{
M. GONZÁLEZ,* \\ M. MOLINA*** AND \\ I. DEL PUERTO, ${ }^{*}$ Universidad de Extremadura
}

\begin{abstract}
In this paper, we investigate the asymptotic behaviour of controlled branching processes with random control functions. In a critical case, we establish sufficient conditions for both their almost-sure extinction and for their nonextinction with a positive probability. For some suitably chosen norming constants, we also determine different kinds of limiting behaviour for this class of processes.
\end{abstract}

Keywords: Branching process; controlled process; extinction probability; limiting behaviour

2000 Mathematics Subject Classification: Primary 60J80

\section{Introduction}

A controlled branching process with random control function is a discrete-time stochastic model of the form

$$
Z_{0}=N, \quad Z_{n+1}=\sum_{j=1}^{\phi_{n}\left(Z_{n}\right)} X_{n j}, \quad n=0,1, \ldots,
$$

where an empty sum is taken to be $0, N$ is a positive integer, and $\left\{X_{n j}, n=0,1, \ldots, j=\right.$ $1,2, \ldots\}$ and $\left\{\phi_{n}(k), n, k=0,1, \ldots\right\}$ are independent sets of nonnegative integer-valued random variables on the same probability space. The variables $X_{n j}$ are independent and identically distributed, their common probability law is called the offspring probability distribution, and, for $n=0,1, \ldots$, the $\left\{\phi_{n}(k)\right\}_{k \geq 0}$ are independent stochastic processes such that $\phi_{n}(k), n=0,1, \ldots$, are identically distributed. Intuitively, $Z_{n}$ represents the size of the $n$th generation of a population under the influence of a random control mechanism. The $\phi_{n}(k), n=0,1, \ldots, k=0,1, \ldots$, will be referred to as control variables.

Several questions concerning this control model have been investigated in [1], [3], [15], and [19]. Recently (see [4], [5], [6], and [7]), it has been studied in the framework of asymptotically linear growth of the mathematical expectations of the control variables. In this paper, we continue the research into this class of branching models and, in a critical case, we investigate some pertinent questions that have not previously been considered in the literature. In Section 2, some notation and working assumptions are given. Sufficient conditions for the almost-sure

Received 27 February 2004; revision received 28 October 2004.

* Postal address: Departamento de Matemáticas, Facultad de Ciencias, Universidad de Extremadura, 06071 Badajoz, Spain.

** Email address: mmolina@unex.es 
extinction or for the existence of a positive probability of nonextinction are provided in Section 3, and Section 4 is devoted to investigating different kinds of limiting behaviour for $\left\{Z_{n}\right\}_{n \geq 0}$, when suitably normed. In particular, gamma, normal, and degenerate laws are obtained as asymptotic distributions. In order to allow for a more comprehensible reading, the proofs are relegated to Section 5 .

\section{Notation and working assumptions}

From (1), by considering the conditions established for the variables $X_{n j}$ and $\phi_{n}(k)$, it is easily verified that $\left\{Z_{n}\right\}_{n \geq 0}$ is a homogeneous Markov chain whose state space is included in the nonnegative integers. For simplicity, we shall assume that the offspring probability distribution, denoted by $\left\{p_{k}\right\}_{k \geq 0}$ with $p_{k}:=\mathrm{P}\left(X_{01}=k\right)$, and the control variables are such that the positive integers form a class of communicating, aperiodic states. Moreover, from now on we will consider controlled branching processes with random control functions such that $\mathrm{P}\left(\phi_{0}(0)=0\right)=1$, i.e. 0 is an absorbent state, and at least one of the following conditions holds:

(i) $p_{0}>0$ or

(ii) $\mathrm{P}\left(\phi_{0}(k)=0\right)>0, k=1,2, \ldots$

Under these assumptions, the classical extinction-explosion duality in branching process theory holds [19], namely $\mathrm{P}\left(Z_{n} \rightarrow 0\right)+P\left(Z_{n} \rightarrow \infty\right)=1$.

Let us write $m:=\mathrm{E}\left[X_{01}\right], \sigma^{2}:=\operatorname{var}\left[X_{01}\right], \varepsilon(k):=\mathrm{E}\left[\phi_{0}(k)\right]$, and $v^{2}(k):=\operatorname{var}\left[\phi_{0}(k)\right], k=$ $0,1, \ldots$, and define $\tau(k)$ as $\mathrm{E}\left[Z_{n+1} Z_{n}^{-1} \mid Z_{n}=k\right], k=1,2, \ldots$, which is equivalent to $m k^{-1} \varepsilon(k)$; this is intuitively interpreted as the expected growth rate per individual when, in a certain generation, there are $k$ individuals. In order to obtain conditions for the almost-sure extinction, different possible behaviours for the sequence $\{\tau(k)\}_{k \geq 1}$, relative to 1 , have been considered in [4]. In particular, the cases $\lim \sup _{k \rightarrow \infty} \tau(k)<1$ and $\liminf _{k \rightarrow \infty} \tau(k)>1$, respectively called subcritical and supercritical, have been investigated. Here, we shall be concerned with the complementary situation, i.e. when

$$
\liminf _{k \rightarrow \infty} \tau(k) \leq 1 \leq \limsup _{k \rightarrow \infty} \tau(k),
$$

which is referred to as the critical case.

Note that $\left\{Z_{n}\right\}_{n \geq 0}$ almost surely satisfies the relation

$$
Z_{n+1}=Z_{n}+g\left(Z_{n}\right)+\xi_{n+1}, \quad n=0,1, \ldots,
$$

where

$$
Z_{0}=N, \quad g(k):=m \varepsilon(k)-k, \quad k=0,1, \ldots, \quad \text { and } \quad \xi_{n+1}:=Z_{n+1}-\mathrm{E}\left[Z_{n+1} \mid Z_{n}\right] .
$$

It is clear that $\left\{\xi_{n}\right\}_{n \geq 1}$ is a zero-mean, square-integrable martingale difference sequence. For simplicity, let us write $\ell_{\delta}(k):=\mathrm{E}\left[\left|\xi_{n+1}\right|^{\delta} \mid Z_{n}=k\right], k=1,2, \ldots, \delta>0$. The conditional second moments depend only on the instantaneous state of the process. In fact, $\ell_{2}(k)=$ $v^{2}(k) m^{2}+\varepsilon(k) \sigma^{2}, k=1,2, \ldots$

Expression (2) can be rewritten, at least in the event $\left\{g\left(Z_{n}\right) \neq 0\right\}$, in the alternative form

$$
Z_{n+1}=Z_{n}+g\left(Z_{n}\right)\left(1+\eta_{n+1}\right), \quad n=0,1, \ldots,
$$

where $\eta_{n+1}:=\xi_{n+1} g\left(Z_{n}\right)^{-1}$. Obviously, $\left\{\eta_{n}\right\}_{n \geq 1}$ is also a zero-mean, square-integrable martingale difference sequence. 
In order to investigate their asymptotic properties, taking into account (2) or (3), the class of critical controlled branching processes with random control function can be treated using some specific methodologies for stochastic difference equations.

\section{Extinction probability}

The process becomes extinct when, for some $n, Z_{n}=0$. Let us denote by

$$
q_{N}:=\mathrm{P}\left(Z_{n} \rightarrow 0 \mid Z_{0}=N\right)
$$

the extinction probability in the case that the process starts with $N$ individuals, $N=1,2, \ldots$. Focusing our attention on those cases in which the limit of $\{\tau(k)\}_{k \geq 1}$ exists and $\lim _{k \rightarrow \infty} \tau(k)=$ 1 , we will mainly distinguish two situations. If the convergence of $\{\tau(k)\}_{k \geq 1}$ to 1 is very rapid (with respect to the conditional variance), the whole process will behave as a true critical process, i.e. almost-sure extinction will follow. On the other hand, if the convergence of $\{\tau(k)\}_{k \geq 1}$ is very slow, the process will develop as a regular supercritical process, having a positive probability of nonextinction.

We now provide sufficient conditions for almost-sure extinction (Theorem 1) and for the existence of a positive probability of nonextinction (Theorem 2).

Theorem 1. Assume that

(i) $\tau(k)=1+o(1)$ with $\tau(k) \geq 1$,

(ii) $\ell_{2+\delta}(k)=o\left(\ell_{2}(k) k^{\delta}\right)$ for some $0<\delta \leq 1$, and

(iii) $\lim \sup _{k \rightarrow \infty} 2 \mathrm{~kg}(k) / \ell_{2}(k)<1$.

Then $q_{N}=1, N=1,2, \ldots$

Theorem 2. Assume that

(i) $\tau(k)=1+o(1)$,

(ii) $\ell_{2+\delta}(k)=o\left(g(k) k^{1+\delta}(\log k)^{-s}\right)$ for some $0<\delta \leq 1$ and $s>1$, and

(iii) $\liminf _{k \rightarrow \infty} 2 k g(k) / \ell_{2}(k)>1$.

Then $q_{N}<1, N=1,2, \ldots$

Remark 1. Under assumption (iii) of Theorem 1, a sufficient condition for assumption (ii) to be satisfied is that $\ell_{2+\delta}(k)=o\left(g(k) k^{1+\delta}\right)$. In fact,

$$
\frac{\ell_{2+\delta}(k)}{\ell_{2}(k) k^{\delta}}=\frac{\ell_{2+\delta}(k)}{g(k) k^{1+\delta}} \frac{k g(k)}{\ell_{2}(k)} .
$$

Hence, by assumption (iii) and the fact that $\ell_{2+\delta}(k)=o\left(g(k) k^{1+\delta}\right)$, we deduce that $\ell_{2+\delta}(k)=$ $o\left(\ell_{2}(k) k^{\delta}\right)$. Analogously, under assumption (iii) of Theorem 2, it can be seen that $\ell_{2+\delta}(k)=$ $o\left(\ell_{2}(k) k^{\delta}(\log k)^{-s}\right)$ is a sufficient condition for assumption (ii) to hold. Finally, the third assumption of each theorem can intuitively be interpreted as a speed condition due to the fact that

$$
\frac{k g(k)}{\ell_{2}(k)}=\frac{\tau(k)-1}{\ell_{2}(k) k^{-2}} .
$$


The previous theorems provide the answer to the extinction problem for the most typical cases in this class of controlled models. Unfortunately, certain situations are not covered by them, for instance the case in which $\lim _{k \rightarrow \infty} 2 k g(k)\left(\ell_{2}(k)\right)^{-1}=1$. In the following theorem, we extend our research in that direction. Some results concerning the extinction time $T:=\inf \left\{n>0: Z_{n}=0\right\}$ are also established.

Theorem 3. Assume that $\phi_{0}(k), k=1,2, \ldots$, have infinitely divisible probability distributions such that

(i) $\tau(k)=1+k^{-1} c, c>0, k=1,2, \ldots$,

(ii) $\ell_{2}(k)=v k+O(1), v>0$, and

(iii) $\sup _{k \geq 1}\left(g_{k}^{1 / k}\right)^{\prime \prime \prime}(1)<\infty$ with $g_{k}(s):=\mathrm{E}\left[s^{\phi_{0}(k)}\right], 0 \leq s \leq 1$.

(Here, a prime denotes differentiation.) Then

(a) if $2 c v^{-1} \leq 1$, we have $q_{N}=1, N=1,2, \ldots$, and

(b) if $2 c v^{-1}<1$, we have

$$
\mathrm{P}(T>n-1) \sim k_{1} n^{2 c v^{-1}-1}, \quad n \rightarrow \infty,
$$

while if $2 c v^{-1}=1$, we have

$$
\mathrm{P}(T>n-1) \sim k_{2}(\log n)^{-1}, \quad n \rightarrow \infty,
$$

for some positive constants $k_{i}, i=1,2$.

Remark 2. According to Kolmogorov's classical extinction time result for critical BienayméGalton-Watson processes $\left\{Y_{n}\right\}_{n \geq 0}$, we know that $\mathrm{P}\left(Y_{n}>0\right) \sim 2\left(\sigma^{2} n\right)^{-1}$. We have proved, for our controlled model $\left\{Z_{n}\right\}_{n \geq 0}$, that $\mathrm{P}\left(Z_{n}>0\right)$ decays to 0 more slowly. This is due to the immigration component: note that, by assumption (i) of Theorem 3, there is expected immigration of individuals in each generation. On the other hand, the results obtained are also in parallel with those ones established by Yanev and Yanev for critical branching processes with random migration stopped at 0 . Under a dominating immigration, they proved a speed of convergence analogous to that established in Theorem 3 and, assuming a dominating emigration, obtained, as is logical, a faster decay rate (see [16] and [17] for details).

\section{Limiting behaviour}

In this section, we shall be concerned with the limiting behaviour of the process when suitably normed, in both the nonextinction situation and the extinction case. First, we consider processes that do not become extinct with a positive probability. We assume that $\tau(k)=$ $1+c k^{\alpha-1}+o\left(k^{\alpha-1}\right)$, where $\alpha<1, c>0$, and $\tau(k)>1$. Consequently, we deduce that $g(k)=m \varepsilon(k)-k=c k^{\alpha}+o\left(k^{\alpha}\right)$. For technical reasons, we shall extend $g$ to a twicecontinuously differentiable function on $\mathbb{R}$ :

$$
g(x)= \begin{cases}c x^{\alpha}+o\left(x^{\alpha}\right) & \text { if } x \geq 0, \\ 0 & \text { otherwise }\end{cases}
$$

We shall denote by $\left\{a_{n}\right\}_{n \geq 0}$ the solution of the deterministic recursive equation

$$
a_{0}=1, \quad a_{n+1}=a_{n}+g\left(a_{n}\right), \quad n=0,1, \ldots
$$




\section{Theorem 4. Assume that}

(i) $\tau(k)=1+c k^{\alpha-1}+o\left(k^{\alpha-1}\right), \alpha<1, c>0$, with $\tau(k)>1$,

(ii) $\ell_{2}(k)=v k^{\beta}+o\left(k^{\beta}\right), \quad \beta \leq \alpha+1, v>0$, and

(iii) $\ell_{2+\delta}(k)=O\left(\left(\ell_{2}(k)\right)^{1+\delta / 2}\right)$ for some $\delta>0$.

The following statements then hold.

(a) If $\beta=\alpha+1$ and $2 c v^{-1}>1$ then, for all real numbers $z$,

$$
\lim _{n \rightarrow \infty} \mathrm{P}\left(n^{-1} Z_{n}^{1-\alpha} \leq z \mid Z_{r} \rightarrow \infty\right)=\Gamma_{a, b}(z),
$$

where $\Gamma_{a, b}$ denotes the gamma distribution function with parameters $a=(v-v \alpha)^{-1}(2 c-v \alpha)$ and $b=2^{-1} v(1-\alpha)^{2}$.

(b) If $0<\alpha<1$ and $\beta<\alpha+1$ then, in the event $\left\{Z_{r} \rightarrow \infty\right\}$,

1. for $\beta<3 \alpha-1$ we have

$$
a_{n}^{-1} Z_{n} \rightarrow 1 \quad \text { almost surely(a.s.), } \quad g\left(a_{n}\right)^{-1}\left(Z_{n}-a_{n}\right) \text { converges a.s., }
$$

as $n \rightarrow \infty$, and

2. for $\beta \geq 3 \alpha-1$ we have $a_{n}^{-1} Z_{n} \stackrel{\mathrm{P}}{\rightarrow} 1$ as $n \rightarrow \infty$ and, for all real numbers $z$,

$$
\lim _{n \rightarrow \infty} \mathrm{P}\left(\Delta_{n}^{-1 / 2} \frac{Z_{n}-a_{n}}{g\left(a_{n}\right)} \leq z \mid Z_{r} \rightarrow \infty\right)=\phi^{*}(z),
$$

where $\phi^{*}$ is the standard normal distribution function and

$$
\Delta_{n}= \begin{cases}v c^{-3}(1-\alpha)^{-1} \log n & \text { if } \beta=3 \alpha-1, \\ v(\beta-3 \alpha+1)^{-1} c^{(\beta-2) /(1-\alpha)}((1-\alpha) n)^{(\beta-3 \alpha+1) /(1-\alpha)} & \text { if } \beta>3 \alpha-1 .\end{cases}
$$

Remark 3. Theorem 4 makes sense because, under its assumptions, Theorem 2 holds and, therefore, $\left\{Z_{n}\right\}_{n \geq 0}$ does not die out with a positive probability. In fact, from assumption (i) it is clear that $\tau(k)=1+o(1)$. Moreover, from (4) and assumption (ii), we can show that

$$
\lim _{k \rightarrow \infty} \frac{2 k g(k)}{\ell_{2}(k)}= \begin{cases}2 c v^{-1} & \text { if } \beta=\alpha+1 \\ \infty & \text { otherwise }\end{cases}
$$

Finally, using assumptions (ii) and (iii), we have, for some positive constant $M$,

$$
\begin{aligned}
\frac{\ell_{2+\delta}(k)}{\ell_{2}(k) k^{\delta}(\log k)^{-s}} & \leq M \frac{\left(\ell_{2}(k)\right)^{\delta / 2}}{k^{\delta}}(\log k)^{s} \\
& =M \frac{\nu^{\delta / 2} k^{\beta \delta / 2}+o\left(k^{\beta \delta / 2}\right)}{k^{\delta}}(\log k)^{s} \\
& =M \nu^{\delta / 2} k^{\delta(\beta-2) / 2}(1+o(1))(\log k)^{s} \rightarrow 0 \text { as } k \rightarrow \infty,
\end{aligned}
$$

and, consequently (see Remark 1$), \ell_{2+\delta}(k)=o\left(g(k) k^{1+\delta}(\log k)^{-s}\right)$. Note that, for $\beta>\alpha+1$ or for $\beta=\alpha+1$ and $2 c v^{-1}<1$, the process becomes extinct with probability 1 (see Theorem 1 ). 


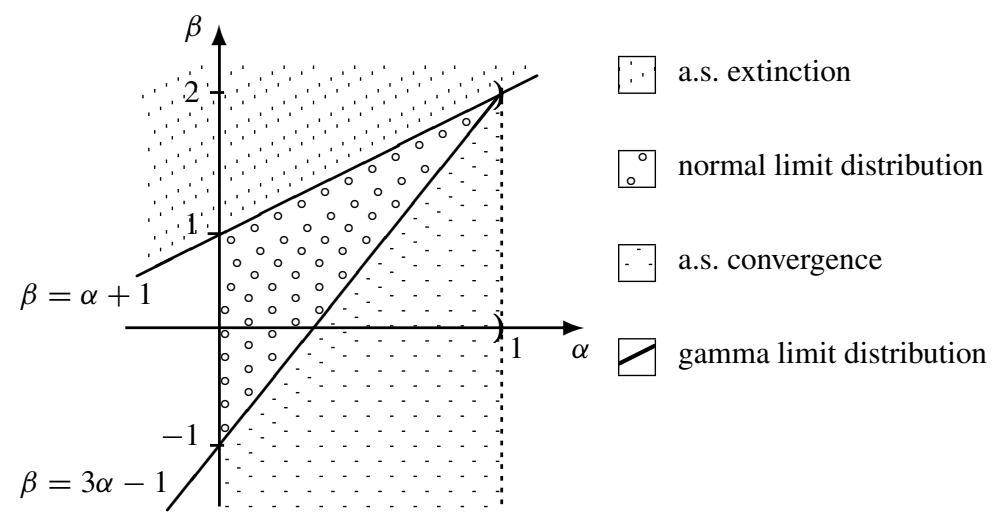

Figure 1.

Figure 1 shows, in a plot in the $(\alpha, \beta)$-plane, a simplified scheme corresponding to the different kinds of limiting behaviour obtained for $\left\{Z_{n}\right\}_{n \geq 0}$, when suitably normed.

Corollary 1. (a) Under the conditions established in Theorem 4(a), for all real numbers $z$,

1. $\lim _{n \rightarrow \infty} \mathrm{P}\left(n^{-1} Z_{n}^{1-\alpha} \leq z\right)=q_{N} \mathbf{1}_{\{z \geq 0\}}+\left(1-q_{N}\right) \Gamma_{a, b}(z)$,

2. $\lim _{n \rightarrow \infty} \mathrm{P}\left(n^{-1} Z_{n}^{1-\alpha} \leq z \mid Z_{n}>0\right)=\Gamma_{a, b}(z)$,

with $a=(v-v \alpha)^{-1}(2 c-v \alpha)$ and $b=2^{-1} v(1-\alpha)^{2}$.

(b) Under the conditions established in Theorem 4(b)2, for all real numbers $z$,

$$
\lim _{n \rightarrow \infty} \mathrm{P}\left(\Delta_{n}^{-1 / 2} \frac{Z_{n}-a_{n}}{g\left(a_{n}\right)} \leq z \mid Z_{n}>0\right)=\phi^{*}(z) .
$$

Remark 4. We can show that $a_{n} \sim(c(1-\alpha) n)^{(1-\alpha)^{-1}}$ as $n \rightarrow \infty$. In fact, because $\alpha<1$ and $c>0,\left\{a_{n}\right\}_{n \geq 0}$ converges to infinity and we can therefore suppose that $a_{n} \neq 0$ for every $n \geq 0$. Taking into account Lemma 1 (in Appendix A) and (4), we have

$$
\begin{aligned}
a_{n+1}^{1-\alpha} & =a_{n}^{1-\alpha}\left(1+\frac{a_{n+1}-a_{n}}{a_{n}}\right)^{1-\alpha} \\
& =a_{n}^{1-\alpha}+(1-\alpha) a_{n}^{-\alpha} g\left(a_{n}\right)+O\left(\frac{g\left(a_{n}\right)^{2}}{a_{n}^{1+\alpha}}\right) \\
& =a_{n}^{1-\alpha}+(1-\alpha) c+o(1) .
\end{aligned}
$$

Hence, we have $a_{n}^{1-\alpha}=c(1-\alpha) n+o(n)$, and the result follows.

Remark 5. From a practical point of view, it is interesting to look for easily checked sufficient conditions which guarantee that the hypotheses about the $(2+\delta)$ th conditional absolute moment of $\xi_{n+1}$ hold. In this sense, we can verify assumption (iii) of Theorem 4 by using both the fact that $|a+b|^{r} \leq C_{r}\left(|a|^{r}+|b|^{r}\right), r>0$, for some positive constant $C_{r}$ (called the $C_{r}$-inequality) 
and the Marcinkiewicz-Zygmund inequality (see Lemma 2 in Appendix A). Doing so, we find that, as $k \rightarrow \infty$,

$$
\ell_{2+\delta}(k) \leq C_{2+\delta}\left(\mathrm{E}\left[\left|\sum_{i=1}^{\phi_{n}(k)}\left(X_{n i}-m\right)\right|^{2+\delta}\right]+m^{2+\delta} \mathrm{E}\left[\left|\phi_{n}(k)-\varepsilon(k)\right|^{2+\delta}\right]\right)
$$

and

$$
\mathrm{E}\left[\left|\sum_{i=1}^{\phi_{n}(k)}\left(X_{n i}-m\right)\right|^{2+\delta}\right]=O\left(\mathrm{E}\left[\left(\phi_{n}(k)\right)^{1+\delta / 2}\right]\right) .
$$

Hence, the conditions

$$
\mathrm{E}\left[\left(\phi_{0}(k)\right)^{1+\delta / 2}\right]=O\left(k^{\beta(1+\delta / 2)}\right) \quad \text { and } \quad \mathrm{E}\left[\left|\phi_{0}(k)-\varepsilon(k)\right|^{2+\delta}\right]=O\left(k^{\beta(1+\delta / 2)}\right)
$$

imply that

$$
\ell_{2+\delta}(k)=O\left(k^{\beta(1+\delta / 2)}\right)
$$

and, as a consequence, that

$$
\ell_{2+\delta}(k)=O\left(\left(\ell_{2}(k)\right)^{1+\delta / 2}\right) .
$$

Under the conditions of Theorem 3, we know that the process becomes extinct with probability 1 . In the following result, we investigate the limiting distribution of $n^{-1} Z_{n}$ conditioned on $\left\{Z_{n}>0\right\}$.

Theorem 5. Under the hypotheses of Theorem 3, if $2 c v^{-1} \leq 1$ then

$$
\lim _{n \rightarrow \infty} \mathrm{P}\left(n^{-1} Z_{n} \leq z \mid Z_{n}>0\right)=\Gamma_{a, b}(z),
$$

with $a=1$ and $b=2^{-1} v$.

Remark 6. Note that, in this situation, the parameter $a$ does not depend on $c$ or $v$, unlike the analogous result in the case in which extinction is not almost sure. This result is similar to the result of Kolmogorov and Yaglom concerning the limiting exponential distribution for the critical Bienaymé-Galton-Watson process. Both models have the same exponential limiting distribution on their nonextinction paths, in spite of the fact that the decay rates of their nonextinction probabilities are different. In particular, if $\phi_{0}(k):=k+Y, k=1,2, \ldots$, where $Y$ is a nonnegative integer-valued random variable independent of $\left\{X_{n j}, n=0,1, \ldots, j=1,2, \ldots\right\}$ and such that $\mathrm{E}[Y]<\infty$, then $\left\{Z_{n}\right\}_{n \geq 0}$ is a Galton-Watson process with immigration. The classical result about the limiting gamma distribution for this process in the critical case (see [10]) is included in Theorem 4. Analogous results have been also obtained for critical branching processes with random migration, both when immigration dominates emigration and vice versa (see [16], [17], and [18]).

\section{Proofs}

In the proofs of Theorems 1 and 2 it will be necessary to consider the following result.

Proposition 1. Let $\left\{X_{n}\right\}_{n \geq 0}$ be a sequence of nonnegative random variables and let $\left\{\mathcal{F}_{n}\right\}_{n \geq 0}$ be a nondecreasing sequence of $\sigma$-algebras such that $X_{n}$ is $\mathcal{F}_{n}$-measurable for each $n$. Let $f$ be a positive function on $\mathbb{R}^{+}$. 
(a) If $f$ is increasing and

$$
\mathrm{E}\left[f\left(X_{n+1}\right) \mid \mathcal{F}_{n}\right] \leq f\left(X_{n}\right) \text { a.s. on }\left\{X_{n}>A\right\}, n=0,1, \ldots,
$$

for some positive constant $A$, then $\mathrm{P}\left(X_{n} \rightarrow \infty\right)=0$.

(b) Suppose that, for any constant $C^{*}$, there exists an $n=1,2, \ldots$ such that $\mathrm{P}\left(X_{n}>C^{*}\right)>0$ and, moreover, that $\mathrm{P}\left(X_{n} \rightarrow 0\right)+\mathrm{P}\left(X_{n} \rightarrow \infty\right)=1$. If $f$ is decreasing and

$$
\mathrm{E}\left[f\left(X_{n+1}\right) \mid \mathcal{F}_{n}\right] \leq f\left(X_{n}\right) \text { a.s. on }\left\{X_{n}>A\right\}, n=0,1, \ldots,
$$

for some positive constant $A$, then $\mathrm{P}\left(X_{n} \rightarrow \infty\right)>0$.

Proof. The proposition is proved using reasoning similar to that used by Kersting in [12].

(a) Taking into account the fact that $\left\{X_{n} \rightarrow \infty\right\}=\bigcup_{j=1}^{\infty}\left\{\inf _{n \geq j} X_{n}>A\right\} \cap\left\{X_{n} \rightarrow \infty\right\}$, it will be sufficient to verify that, for every $j \geq 1$,

$$
\mathrm{P}\left(\left\{\inf _{n \geq j} X_{n}>A\right\} \cap\left\{X_{n} \rightarrow \infty\right\}\right)=0 .
$$

For an arbitrary $j \geq 1$, let us define

$$
T(A):= \begin{cases}\inf \left\{n \geq j: X_{n} \leq A\right\} & \text { if } \inf _{n \geq j} X_{n} \leq A \\ \infty & \text { otherwise }\end{cases}
$$

We consider the sequence $\left\{Y_{n}\right\}_{n \geq 0}$, where $Y_{n}:=X_{T(A) \wedge(j+n)}$. After some calculations, we find that

$$
\mathrm{E}\left[Y_{n+1} \mid \mathcal{F}_{j+n}\right] \leq Y_{n} \quad \text { a.s., } \quad n=0,1, \ldots
$$

Therefore, $\left\{Y_{n}\right\}_{n \geq 0}$ is a nonnegative supermartingale with respect to $\left\{\mathcal{F}_{j+n}\right\}_{n \geq 0}$ and, from the martingale convergence theorem (see, e.g. [2, p. 246]), it follows that $\left\{Y_{n}\right\}_{n \geq 0}$ is almost surely convergent to a random variable $Y$ such that $\mathrm{P}(0 \leq Y<\infty)=1$. It now follows that, almost surely,

$$
Y= \begin{cases}X_{T(A)} & \text { if } \inf _{n \geq j} X_{n} \leq A \\ \lim _{n \rightarrow \infty} X_{j+n} & \text { otherwise }\end{cases}
$$

and we obtain (5).

(b) Let $Y_{n}^{*}:=f\left(X_{n}\right) \wedge f(A), n=0,1, \ldots$ It can be shown that

$$
\mathrm{E}\left[Y_{n+1}^{*} \mid \mathcal{F}_{n}\right] \leq \mathrm{E}\left[f\left(X_{n+1}\right) \mid \mathcal{F}_{n}\right] \wedge f(A) \leq Y_{n}^{*} \quad \text { a.s., } \quad n=0,1, \ldots
$$

Hence, $\left\{Y_{n}^{*}\right\}_{n \geq 0}$ is a nonnegative supermartingale with respect to $\left\{\mathcal{F}_{n}\right\}_{n \geq 0}$ and, again using the martingale convergence theorem, it is almost surely convergent to a finite, nonnegative random variable $Y^{*}$. Moreover, because $\left\{Y_{n}^{*}\right\}_{n \geq 0}$ is bounded, it follows that it is also convergent in $L^{1}$. Suppose that $\mathrm{P}\left(X_{n} \rightarrow \infty\right)=0$; then $\mathrm{P}\left(X_{n} \rightarrow 0\right)=1$ and it follows that $\mathrm{E}\left[Y^{*}\right]=f(A)$. Since $\left\{\mathrm{E}\left[Y_{n}^{*}\right]\right\}_{n \geq 0}$ is decreasing, $\mathrm{E}\left[Y_{n}^{*}\right] \geq f(A), n=0,1, \ldots$ Now, $Y_{n}^{*} \leq f(A), n=0,1, \ldots$; hence, it follows, for every $n$, that $Y_{n}^{*}=f(A)$ almost surely and, since $f$ is decreasing, that $X_{n} \leq A$ almost surely, $n=0,1, \ldots$. This contradicts the first assumption in Proposition 1(b). 


\subsection{Proof of Theorem 1}

For $x>-1$ and $0 \leq \delta \leq 1$, the following inequality holds:

$$
\log (x+1) \leq x-\frac{1}{2} x^{2}+\frac{1}{2}|x|^{2+\delta} .
$$

From (2), we have

$$
\log \left(Z_{n+1}+1\right)=\log \left(Z_{n}+1\right)+\log \left(1+\frac{g\left(Z_{n}\right)}{Z_{n}+1}+\frac{\xi_{n+1}}{Z_{n}+1}\right) .
$$

Hence, using (6) with $x=\left(g\left(Z_{n}\right)+\xi_{n+1}\right) /\left(Z_{n}+1\right)$, taking expectations, applying the $C_{r}$-inequality, and using the fact that, by assumption (i) (of Theorem 1 ), $g(k)=k(\tau(k)-1) \geq 0$, for a certain positive constant $C_{2+\delta}$ we obtain

$$
\begin{aligned}
\mathrm{E}\left[\log \left(Z_{n+1}+1\right) \mid Z_{n}=k\right] \leq & \log (k+1)+\frac{g(k)}{k}-\frac{(g(k))^{2}}{2(k+1)^{2}}-\frac{\ell_{2}(k)}{2(k+1)^{2}} \\
& +\frac{1}{2} C_{2+\delta}\left(\frac{(g(k))^{2+\delta}}{k^{2+\delta}}+\frac{\ell_{2+\delta}(k)}{k^{2+\delta}}\right) \\
= & \log (k+1)+\frac{g(k)}{k} \Delta_{1}(k)-\frac{\ell_{2}(k)}{2 k^{2}} \Delta_{2}(k), \quad k=1,2, \ldots,
\end{aligned}
$$

where

$$
\Delta_{1}(k)=1-\frac{g(k) k}{2(k+1)^{2}}+\frac{1}{2} C_{2+\delta} \frac{(g(k))^{1+\delta}}{k^{1+\delta}}
$$

and

$$
\Delta_{2}(k)=\frac{k^{2}}{(k+1)^{2}}-\frac{1}{2} C_{2+\delta} \frac{\left(\ell_{2+\delta}(k)\right)^{1+\delta}}{\ell_{2}(k) k^{\delta}} .
$$

From assumptions (i) and (ii), we deduce that $\lim _{k \rightarrow \infty} \Delta_{1}(k)=\lim _{k \rightarrow \infty} \Delta_{2}(k)=1$, so, for $k$ sufficiently large and $\epsilon>0$ sufficiently small, we have

$$
\frac{g(k)}{k} \Delta_{1}(k)-\frac{\ell_{2}(k)}{2 k^{2}} \Delta_{2}(k) \leq \frac{g(k)}{k}(1+\epsilon)-\frac{\ell_{2}(k)}{2 k^{2}}(1-\epsilon) .
$$

Now, from assumption (iii), if we choose an $\epsilon$ such that $0<\epsilon<(1-b)(1+b)^{-1}$ with $b:=\lim \sup _{k \rightarrow \infty} 2 k g(k) / \ell_{2}(k)$, then the right-hand side of the previous inequality is negative. Therefore, it follows that if $Z_{n} \geq A$, for some sufficiently large constant $A$, then

$$
\mathrm{E}\left[\log \left(Z_{n+1}+1\right) \mid \mathcal{F}_{n}\right] \leq \log \left(Z_{n}+1\right) \quad \text { a.s. }
$$

where $\mathcal{F}_{n}$ denotes the $\sigma$-algebra generated by $Z_{0}, \ldots, Z_{n}$. The result then follows by applying Proposition 1(a).

\subsection{Proof of Theorem 2}

Let $f(x)=(\log x)^{-\alpha}, x>0, \alpha \leq s-1$. It was shown in [12] that, for $x+h \geq 3, x \geq 3$, and a sufficiently large constant $C^{*}$,

$$
f(x+h) \leq f(x)+f^{\prime}(x) h+\frac{1}{2} f^{\prime \prime}(x) h^{2}+C^{*} \frac{|h|^{2+\delta}}{(\log x)^{1+\alpha} x^{2+\delta}}+\mathbf{1}_{\{h \leq-x / 2\}} .
$$


From (2), setting $x=Z_{n}+3$ and $h=g\left(Z_{n}\right)+\xi_{n+1}$, applying Chebychev's conditional equality and the $C_{r}$-inequality, and taking expectations, we find that

$$
\begin{aligned}
\mathrm{E}\left[f\left(Z_{n+1}+3\right) \mid Z_{n}=k\right] \leq & f(k+3)+f^{\prime}(k+3) g(k)+\frac{1}{2} f^{\prime \prime}(k+3)\left(g(k)^{2}+\ell_{2}(k)\right) \\
& +C_{1}\left(\frac{(g(k))^{2+\delta}}{(\log k)^{1+\alpha} k^{2+\delta}}+\frac{\ell_{2+\delta}(k)}{(\log k)^{1+\alpha} k^{2+\delta}}\right) \\
& +C_{2} \frac{\ell_{2+\delta}(k)}{k^{2+\delta}}, \quad k=1,2, \ldots,
\end{aligned}
$$

for some positive constants $C_{i}, i=1,2$. By assumptions (i) and (ii) (of Theorem 2), and using reasoning similar to that used in the proof of Theorem 1, we find that, for $k$ sufficiently large and $\epsilon>0$ sufficiently small,

$$
\mathrm{E}\left[f\left(Z_{n+1}+3\right) \mid Z_{n}=k\right] \leq f(k+3)-\frac{\alpha g(k)}{(\log k)^{1+\alpha} k}(1-\epsilon)+\frac{\alpha \ell_{2}(k)}{2(\log k)^{1+\alpha} k^{2}}(1+\epsilon)
$$

and, from assumption (iii), we deduce that if $Z_{n}$ is sufficiently large, then

$$
\mathrm{E}\left[f\left(Z_{n+1}+3\right) \mid \mathcal{F}_{n}\right] \leq f\left(Z_{n}+3\right) \quad \text { a.s., }
$$

where, as in Theorem $1, \mathcal{F}_{n}$ is the $\sigma$-algebra generated by $Z_{0}, \ldots, Z_{n}$. Application of Proposition 1(b) completes the proof.

\subsection{Proof of Theorem 3}

We will need the following preliminary result.

Proposition 2. Under the hypotheses of Theorem 3, there exist continuous and nondecreasing functions $A_{i}, a_{i}:[0,1] \rightarrow[0,1], i=1,2$, that have a continuous derivative on a small left-neighbourhood of 1 and satisfy the following conditions.

(a) $A_{i}(1)=a_{i}(1)=1, A_{i}(0)>0, A_{i}^{\prime}(1)=c>0$, and $a_{i}^{\prime}(1)=1$, for $i=1,2$.

(b) For $i=1,2, a_{i}(s)>s, 0 \leq s<1$, and $a_{i}(s)$ has continuous second derivative on a small left-neighbourhood of 1 , with $a_{i}^{\prime \prime}(1)=v>0$.

(c) There exist nonincreasing functions $E_{i}$ and $e_{i}, i=1,2$, on $(0,1)$, such that

$$
\left|\frac{a_{i}(s)-s}{(1-s)^{2}}-\frac{v}{2}\right| \leq e_{i}(s), \quad\left|\frac{1-A_{i}(s)}{1-s}-c\right| \leq E_{i}(s), \quad i=1,2,
$$

and

$$
\int_{\eta_{i}}^{1} \frac{E_{i}(s)}{1-s} \mathrm{~d} s<\infty, \quad \int_{\eta_{i}}^{1} \frac{e_{i}(s)}{1-s} \mathrm{~d} s<\infty, \quad \text { for some } \eta_{i} \in(0,1), i=1,2 .
$$

(d) $A_{1}(s)\left(a_{1}(s)\right)^{k} \leq h_{k}(s) \leq A_{2}(s)\left(a_{2}(s)\right)^{k}, 0 \leq s \leq 1, k=1,2, \ldots$, where $h_{k}(s):=$ $\mathrm{E}\left[s^{Z_{n+1}} \mid Z_{n}=k\right]$.

Proof. Since $\phi_{0}(k)$ has infinitely divisible probability distribution, it follows that, for each $j \geq 1$, there exists a probability generating function $f_{k j}$ (with associated probability distribution belonging to the same family of probability distributions as that of $\phi_{0}(k)$ ), such that 
$g_{k}(s)=\left(f_{k j}(s)\right)^{j}, 0 \leq s \leq 1$. Let us consider the sequence of probability generating functions $\left\{l_{k}\right\}_{k \geq 0}$, where $l_{k}(s):=f_{k k}(f(s)), 0 \leq s \leq 1$, with $f(s):=\mathrm{E}\left[s^{X_{01}}\right]$. It is easy to verify that $h_{k}(s)=g_{k}(f(s))=\left(l_{k}(s)\right)^{k}$. Let

$$
m_{k}:=l_{k}^{\prime}(1) \quad \text { and } \quad \pi_{k}:=l_{k}^{\prime \prime}(1), \quad k=1,2, \ldots
$$

Taking into account the results of [8] and the existence of the functions $A_{i}$ and $a_{i}, i=1,2$, parts (a)-(d) are guaranteed to hold if

(p1) $m_{k}=1+k^{-1} \delta, k=1,2, \ldots$, for some $\delta>0$,

(p2) $\left|\pi_{k}-\mu\right| \leq k^{-1} M, k=1,2, \ldots$, for some $M>0$ and $\mu>0$, and

(p3) $\sup _{k \geq 1} \sum_{j=0}^{\infty} j^{2+r} \pi_{k j}<\infty$ for some $r>0$, where $\left\{\pi_{k j}\right\}_{j \geq 0}$ denotes the probability distribution associated with $l_{k}$.

Now,

$$
m_{k}=k^{-1} \varepsilon(k) m=1+k^{-1} c, \quad k=1,2, \ldots,
$$

from which (p1) follows. Using assumptions (i) and (ii) (of Theorem 3), we have

$$
\pi_{k}=k^{-1} \ell_{2}(k)+(\tau(k))^{2}-\tau(k)=v+k^{-1}\left(O(1)+c\left(1+k^{-1} c\right)\right), \quad k=1,2, \ldots,
$$

and, consequently, (p2) follows with $\mu=v$. By using assumptions (i) and (ii) again, we deduce that $(v(k))^{2}=O(k)$,

Finally, we have

$$
l_{k}^{\prime \prime \prime}(1)=3 m f^{\prime \prime}(1) f_{k k}^{\prime \prime}(1)+m^{3} f_{k k}^{\prime \prime \prime}(1)+f^{\prime \prime \prime}(1) f_{k k}^{\prime}(1) .
$$

Now, by assumptions (i) and (ii), we obtain

$$
f^{\prime \prime \prime}(1) f_{k k}^{\prime}(1)=f^{\prime \prime \prime}(1) k^{-1} \varepsilon(k)=O(1)
$$

and

$$
f_{k k}^{\prime \prime}(1)=k^{-2}\left(k g_{k}^{\prime \prime}(1)-(k-1)\left(g_{k}^{\prime}(1)\right)^{2}\right)=O(1) .
$$

On the other hand, by assumption (iii), we have $f_{k k}^{\prime \prime \prime}(1)=\left(g_{k}^{1 / k}\right)^{\prime \prime \prime}(1)=O(1)$ and, therefore, (p3) holds.

We are now ready to prove Theorem 3. Part (a) follows from Proposition 2 if we apply Theorem 2.1(a) of [8]. To prove part (b), let $A_{i}$ and $a_{i}, i=1,2$, be the functions provided in Proposition 2 and, for $j=0,1, \ldots$, let us denote by $a_{i}^{(j)}$ the $j$ th functional iterate of $a_{i}$, i.e. $a_{i}^{(j)}(s)=a_{i}\left(a_{i}^{(j-1)}(s)\right)$ with $a_{i}^{(0)}(s)=s$. Under the conditions satisfied by $A_{i}$ and $a_{i}$, by Lemma 3.3 of [8] we have

$$
\prod_{j=0}^{n-1} A_{i}\left(a_{i}^{(j)}(0)\right) \sim n^{-2 c v^{-1}} M_{i}, \quad i=1,2,
$$

where $M_{i}, i=1,2$, are positive constants. Therefore,

$$
\sum_{n=0}^{\infty} \prod_{j=0}^{n-1} A_{i}\left(a_{i}^{(j)}(0)\right)=\infty, \quad i=1,2,
$$

and application of Lemmas 2.1 and 2.2 of [9] completes the proof. 


\subsection{Proof of Theorem 4}

Let us introduce the function

$$
G(x):=\int_{1}^{x} \frac{1}{g(z)} \mathrm{d} z, \quad x \geq 1 .
$$

Using $g(x)=c x^{\alpha}+o\left(x^{\alpha}\right), x>0$, we can argue that

$$
g^{\prime}(x)=c \alpha x^{\alpha-1}+o\left(x^{\alpha-1}\right) \quad \text { and } \quad G(x) \sim(c(1-\alpha))^{-1} x^{1-\alpha}, \quad x \rightarrow \infty .
$$

Proof of part (a). From (7), we have

$$
\lim _{x \rightarrow \infty} g^{\prime}(x) G(x)=(1-\alpha)^{-1}-1
$$

and, by assumption (ii) (of Theorem 4) and (7), we obtain

$$
\lim _{k \rightarrow \infty} \frac{\ell_{2}(k)}{(g(k))^{2} G(k)}=c^{-1} v(1-\alpha)>0 .
$$

For simplicity, let $\lambda=(1-\alpha)^{-1}$ and $\gamma=c^{-1} v(1-\alpha)$. Taking into account the fact that $2 c v^{-1}>1$, we deduce that $\gamma \lambda<2$. Therefore, the assumptions of Theorem 1 of [13] are satisfied, and we find that, for all real numbers $z$,

$$
\lim _{n \rightarrow \infty} \mathrm{P}\left(n^{-1} G\left(Z_{n}\right) \leq z \mid Z_{r} \rightarrow \infty\right)=\Gamma_{2 / \gamma-\lambda+1, \gamma / 2}(z) .
$$

Now, by (7), in the event $\left\{Z_{r} \rightarrow \infty\right\}$ we have

$$
c^{-1} \lambda Z_{n}^{1-\alpha} G\left(Z_{n}\right)^{-1} \rightarrow 1 \quad \text { a.s., as } n \rightarrow \infty .
$$

Finally, from (8) and (9), Slutsky's theorem, and some properties of the gamma distribution, the result is obtained.

Proof of part (b). In view of relation (3), this part will be proved using Theorem 3 of [11]. Notice that, from assumption (iii),

$$
\mathrm{E}\left[\left|\eta_{n+1}\right|^{2+\delta} \mid Z_{n}=k\right]=O\left(\left(\varphi^{2}(k)\right)^{1+\delta / 2}\right),
$$

where $\varphi^{2}(k):=\mathrm{E}\left[\eta_{n+1}^{2} \mid Z_{n}=k\right]=\ell_{2}(k)(g(k))^{-2}$.

Let us consider the function

$$
\psi(x):=\int_{1}^{x} \frac{\varphi^{2}(z)}{g(z)} \mathrm{d} z, \quad x \geq 1 .
$$

By assumption (ii) and the fact that $g(x)=c x^{\alpha}+o\left(x^{\alpha}\right)$, it follows that

$$
\frac{\varphi^{2}(x)}{g(x)} \sim v c^{-3} x^{\beta-3 \alpha}, \quad x \rightarrow \infty,
$$

and, therefore, in the same limit, that

$$
\psi(x) \sim \begin{cases}v c^{-3}(\beta-3 \alpha+1)^{-1}\left(x^{\beta-3 \alpha+1}-1\right) & \text { if } \beta \neq 3 \alpha-1 \\ v c^{-3} \log x & \text { if } \beta=3 \alpha-1\end{cases}
$$


Since $G(x) \sim(c(1-\alpha))^{-1} x^{1-\alpha}$, we have $G^{-1}(x) \sim(c(1-\alpha) x)^{(1-\alpha)^{-1}}$, where $G^{-1}$ denotes the inverse function of $G$. Consequently, if $\widehat{\psi}(x):=\psi\left(G^{-1}(x)\right)$ then

$$
\widehat{\psi}(x) \sim \begin{cases}v c^{-3}(\beta-3 \alpha+1)^{-1}\left((c(1-\alpha) x)^{(\beta-3 \alpha+1) /(1-\alpha)}-1\right) & \text { if } \beta \neq 3 \alpha-1, \\ v c^{-3}(1-\alpha)^{-1} \log x & \text { if } \beta=3 \alpha-1 .\end{cases}
$$

The following statements now hold.

(A) The function $g$ is positive, twice differentiable on $\mathbb{R}^{+}$, and ultimately concave, and $g^{\prime}$ is ultimately convex.

(B) The function $\varphi^{2}$ is positive and continuously differentiable. Furthermore,

$$
\int_{1}^{x} z^{-2} \varphi^{2}\left(G^{-1}(z)\right) \mathrm{d} z \sim \int_{1}^{x} \nu c^{-2}(c(1-\alpha))^{(\beta-2 \alpha) /(1-\alpha)} z^{(\beta-2 \alpha) /(1-\alpha)-2} \mathrm{~d} z
$$

and, since $\beta<\alpha+1$, the integral

$$
v c^{-2}(c(1-\alpha))^{(\beta-2 \alpha) /(1-\alpha)} \int_{1}^{\infty} z^{(\beta-2) /(1-\alpha)} \mathrm{d} z
$$

is finite. Moreover, $\varphi^{2}\left(G^{-1}(x)\right) \sim v c^{-2}(c(1-\alpha) x)^{(\beta-2 \alpha) /(1-\alpha)}$, so it is ultimately concave or convex.

(C) If $\beta \geq 3 \alpha-1$ then, from (11), we obtain $\psi(\infty)=\infty$ and we also deduce that

$$
\left|g^{\prime \prime}(x) g(x) / \varphi^{2}(x)\right|=O\left(x^{4 \alpha-\beta-2}\right),
$$

which is ultimately decreasing because $4 \alpha-\beta-2<0$. On the other hand, if $\beta<3 \alpha-1$ then, again using (11), $\psi(\infty)<\infty$ and $\left|g^{\prime \prime}(x) g(x)\right|=O\left(x^{2(\alpha-1)}\right)$, which is ultimately decreasing since $\alpha-1<0$.

(D) For $\beta \geq 3 \alpha-1$, we have

$$
g^{\prime}(x)(\psi(x))^{1 / 2}= \begin{cases}O\left(x^{(\beta-(\alpha+1)) / 2}\right) & \text { if } \beta>3 \alpha-1 \\ O\left((\log x)^{1 / 2} x^{\alpha-1}\right) & \text { if } \beta=3 \alpha-1\end{cases}
$$

while, for $\beta<3 \alpha-1$, we have

$$
g^{\prime}(x)(\psi(x))^{1 / 2} \sim \alpha\left(\frac{\nu\left(1-x^{\beta-3 \alpha+1}\right)}{c(3 \alpha-1-\beta)}\right)^{1 / 2} x^{\alpha-1} .
$$

Hence, $g^{\prime}(x)(\psi(x))^{1 / 2} \rightarrow 0$ as $x \rightarrow \infty$.

Considering (10), (12), and statements (A)-(D), the assumptions of Theorem 3 of [11] can be verified. Using the second part of this theorem, we obtain the result of part (b)1 of our Theorem 4 and, from its first part, we find that

$$
a_{n}^{-1} Z_{n} \stackrel{\mathrm{P}}{\rightarrow} 1 \text { in the event }\left\{Z_{r} \rightarrow \infty\right\}
$$

for $\beta \geq 3 \alpha-1$, and that

$$
\lim _{n \rightarrow \infty} \mathrm{P}\left((\widehat{\psi}(n))^{-1 / 2} \frac{Z_{n}-a_{n}}{g\left(a_{n}\right)} \leq z \mid Z_{r} \rightarrow \infty\right)=\phi^{*}(z),
$$


for all real numbers $z$. (Recall that $\phi^{*}$ denotes the standard normal distribution function.) Finally, using Slutsky's theorem and taking into account the fact that

$$
\widehat{\psi}(n) \sim \begin{cases}\frac{v}{c^{3}(1-\alpha)} \log n & \text { if } \beta=3 \alpha-1, \\ \frac{v}{\beta-3 \alpha+1} c^{(\beta-2) /(1-\alpha)}((1-\alpha) n)^{(\beta-3 \alpha+1) /(1-\alpha)} & \text { if } \beta>3 \alpha-1,\end{cases}
$$

we deduce the result of part (b)2.

\subsection{Proof of Corollary 1}

(a) For simplicity, let us consider $Y_{n}=n^{-1} Z_{n}^{1-\alpha}$ and take $z>0$. Then

$$
\begin{aligned}
\mathrm{P}\left(Y_{n} \leq z\right)= & \mathrm{P}\left(Y_{n} \leq z \mid Z_{r} \rightarrow 0\right) \mathrm{P}\left(Z_{r} \rightarrow 0\right) \\
& +\mathrm{P}\left(Y_{n} \leq z \mid Z_{r} \rightarrow \infty\right) \mathrm{P}\left(Z_{r} \rightarrow \infty\right)
\end{aligned}
$$

and, using the fact that $\lim _{n \rightarrow \infty} \mathrm{P}\left(Y_{n} \leq z \mid Z_{r} \rightarrow 0\right)=1$, the fact that $Z_{0}=N$, and Theorem 4(a), we deduce that

$$
\lim _{n \rightarrow \infty} \mathrm{P}\left(Y_{n} \leq z\right)=q_{N}+\Gamma_{a, b}(z)\left(1-q_{N}\right) .
$$

Consequently, part (a) 1 of Corollary 1 holds.

On the other hand, we have

$$
\mathrm{P}\left(Y_{n} \leq z, Z_{n}>0\right)=\mathrm{P}\left(Y_{n} \leq z, Z_{n}>0, Z_{r} \rightarrow \infty\right)+\mathrm{P}\left(Y_{n} \leq z, Z_{n}>0, Z_{r} \rightarrow 0\right) .
$$

Now,

$$
\begin{gathered}
\mathrm{P}\left(Y_{n} \leq z, Z_{n}>0, Z_{r} \rightarrow \infty\right)=\mathrm{P}\left(Y_{n} \leq z, Z_{r} \rightarrow \infty\right), \\
\mathrm{P}\left(Y_{n} \leq z, Z_{n}>0, Z_{r} \rightarrow 0\right) \leq \mathrm{P}\left(Z_{n}>0, Z_{r} \rightarrow 0\right),
\end{gathered}
$$

and, since $\lim _{n \rightarrow \infty} \mathrm{P}\left(Z_{n}>0\right)=\mathrm{P}\left(Z_{r} \rightarrow \infty\right)$ and $\lim _{n \rightarrow \infty} \mathrm{P}\left(Z_{n}>0, Z_{r} \rightarrow 0\right)=0$, by Theorem 4(a), part (a) 2 of Corollary 1 is deduced.

(b) The proof of part (b) is similar to that of part (a)2, taking $Y_{n}=\Delta_{n}^{-1 / 2}\left(g\left(a_{n}\right)\right)^{-1}\left(Z_{n}-a_{n}\right)$ and using Theorem 4(b).

\subsection{Proof of Theorem 5}

Theorem 5 is easily proved using Proposition 2 and applying Lemma 2.3 of [9].

\section{Appendix A. Lemmas}

Lemma 1. For any $0 \leq k \leq 2$ and $x \geq-1$, we have

$$
(1+x)^{k}=1+k x+O\left(x^{2}\right) .
$$

Lemma 2. (Marcinkiewicz-Zygmund inequality.) If $\left\{X_{n}\right\}_{n \geq 1}$ is a sequence of independent and identically distributed random variables with $\mathrm{E}\left[X_{1}\right]=0$ and $\mathrm{E}\left[\left|X_{1}\right|^{p}\right]<\infty, p \geq 2$, then $\mathrm{E}\left[\left|\sum_{j=1}^{n} X_{j}\right|^{p}\right]=O\left(n^{p / 2}\right)$.

We refer the reader to the theorem of [14] for the proof of Lemma 1 and to [2, p. 387] for the proof of Lemma 2. 


\section{Acknowledgements}

We would like to thank the anonymous referees for their constructive comments, interesting suggestions, and useful remarks, which have improved the paper. This research was supported by the Ministerio de Ciencia y Tecnología and the FEDER through the Plan Nacional de Investigación Científica, Desarrollo e Innovación Tecnológica, grant BFM2003-06074.

\section{References}

[1] Bruss, F. T. (1980). A counterpart of the Borel-Cantelli lemma. J. Appl. Prob. 17, 1094-1101.

[2] Chow, Y. S. And Teicher, H. (1997). Probability Theory. Independence, Interchangeability, Martingales, 3rd edn. Springer, New York.

[3] Dion, J.-P. And EssebBar, B. (1995). On the statistics of controlled branching processes. In Branching Processes (Lecture Notes Statist. 99), ed. C. C. Heyde, Springer, New York, pp. 14-21.

[4] González, M., Molina, M. and del Puerto, I. (2002). On the controlled Galton-Watson process with random control function. J. Appl. Prob. 39, 804-815.

[5] González, M., Molina, M. And del Puerto, I. (2003). On the geometric growth in controlled branching processes with random control function. J. Appl. Prob. 40, 995-1006.

[6] González, M., Molina, M. And del Puerto, I. (2004). Limiting distribution for subcritical controlled branching processes with random control function. Statist. Prob. Lett. 67, 277-284.

[7] González, M., Molina, M. and del Puerto, I. (2005). On $L^{2}$-convergence of controlled branching processes with random control function. Bernoulli 11, 37-46.

[8] Höpfner, R. (1985). On some classes of population-size-dependent Galton-Watson processes. J. Appl. Prob. 22, 25-36.

[9] Höpfner, R. (1986). Some results on population-size-dependent Galton-Watson processes. J. Appl. Prob. 23, 297-306.

[10] Jagers, P. (1975). Branching Processes with Biological Applications. John Wiley, London.

[11] Keller, G., Kersting, G. AND Rösler, U. (1987). On the asymptotic behaviour of discrete time stochastic growth processes. Ann. Prob. 15, 305-343.

[12] Kersting, G. (1986). On recurrence and transience of growth models. J. Appl. Prob. 23, 614-625.

[13] Kersting, G. (1992). Asymptotic Г-distribution for stochastic difference equations. Stoch. Process. Appl. 40, $15-28$.

[14] Klebaner, F. (1989). Stochastic difference equations and generalized gamma distributions. Ann. Prob. 17, 178-188.

[15] NaKagawa, T. (1994). The $L^{\alpha}(1<\alpha \leq 2)$ convergence of a controlled branching process in a random environment. Bull. Gen. Ed. Dokkyo Univ. School Medicine 17, 17-24.

[16] Yanev, G. P. AND Yanev, N. M. (1995). Critical branching process with random migration. In Branching Processes (Lecture Notes Statist. 99), ed. C. C. Heyde, Springer, New York, pp. 36-46.

[17] Yanev, G. P. And Yanev, N. M. (2004). A critical branching process with stationary-limiting distribution. Stoch. Anal. Appl. 22, 721-738.

[18] Yanev, G. P., Mitov, K. V. And Yanev, N. M. (2003). Critical branching regenerative process with random migration. J. Appl. Statist. Sci. 12, 41-54.

[19] Yanev, N. M. (1976). Conditions for degeneracy of $\phi$-branching processes with random $\phi$. Theory Prob. Appl. 20, 421-428. 